\title{
Avaliação farmacognóstica de geoprópolis de Melipona fasciculata Smith da Baixada maranhense, Brasil
}

\author{
Richard Pereira Dutra, ${ }^{1}$ Alexandre Michel Costa Nogueira, ${ }^{1}$ Rodrigo René de Oliveira \\ Marques, ${ }^{1}$ Maria Célia Pires Costa, ${ }^{2}$ Maria Nilce Sousa Ribeiro ${ }^{* 1}$ \\ ${ }^{1}$ Laboratório Farmacognosia, Universidade Federal do Maranhão, Campus Bacanga, 65085-580 \\ São Luís-MA, Brasil, \\ ${ }^{2}$ Departamento de Química e Biologia, Universidade Estadual do Maranhão, Campus Universitário Paulo VI, \\ 65055-970 São Luís-MA, Brasil
}

\begin{abstract}
RESUMO: Melipona fasciculata Smith é uma abelha nativa, social e sem ferrão que produz cera, mel e geoprópolis. O presente trabalho objetivou caracterizar, do ponto de vista farmacognóstico, o geoprópolis de Melipona fasciculata, coletado em meliponários nos municípios de Arari, São Bento e São João Batista, da Baixada maranhense, no Estado do Maranhão, visando estabelecer dados para o controle de qualidade e padronização do produto. A metodologia utilizada constou de análises sensoriais, abordagem química, perfis cromatográficos e determinação dos teores de flavonóides. Os resultados demonstram que o geoprópolis apresenta características sensoriais similares às encontradas para própolis de Apis mellifera, presença de compostos fenólicos em maior concentração, além de substâncias da classe dos terpenos e saponinas e ausência de alcalóides. Os perfis cromatográficos indicaram que a composição e concentração das substâncias químicas são diferentes entre as amostras. Os teores de flavonóides variaram de $0,17-2,6 \%$, os quais para a maioria das amostras apresentaram valores acima do mínimo exigido pela legislação brasileira para própolis de Apis mellifera. Os dados encontrados sugerem que as variações qualitativa e quantitativa de flavonóides e outros constituintes químicos no geoprópolis são fortemente afetados pela flora visitada pelas abelhas, região geográfica e fatores ambientais.
\end{abstract}

Unitermos: Geoprópolis, Melipona fasciculata, flavonóides, controle de qualidade, padronização.

\begin{abstract}
Pharmacognostic evaluation of geopropolis of Melipona fasciculata Smith from Baixada Maranhense, Brazil”. Melipona fasciculata Smith is a native, social, stingless bee species that produces wax, honey and geopropolis. This work aimed to do a pharmacological evaluation of Melipona fasciculata geopropolis, collected in beehives in the municipal districts of Arari, São Bento and São João Batista, in Baixada maranhense, in the state of Maranhão, Brazil, seeking to obtain basis for the quality control and standardization of the product. The methodology used included the accomplishment of sensorial analyses, chemical approach, chromatographic profiles and determination of the flavonoids contents. The results demonstrate that the geopropolis have organoleptic characteristics similar to the ones found for the propolis of Apis mellifera, presence of phenolic compounds in larger concentration, besides substances of the class of the terpenes and saponins and absence of alkaloids. The chromatographic profiles indicated that the composition and concentration of the chemical substances are different among the samples. The flavonoids contents ranged between $0.17-2.6 \%$. The content for most of the samples presented values above the minimum demanded by the Brazilian legislation for propolis of Apis mellifera. The found data suggest that the qualitative and quantitative variations of flavonoids and other chemical substances in geopropolis are strongly affected for the flora visited by the bees, geographical area and environmental factors.
\end{abstract}

Keywords: Geopropolis, Melipona fasciculata, flavonoids, control quality, standartization.

\section{INTRODUÇÃO}

Os meliponíneos, abelhas nativas, sociais e sem ferrão, ocupam grande parte das regiões de clima tropical no planeta, especialmente na América do Sul. No Brasil são conhecidas mais de 400 espécies de abelhas nativas, as quais são responsáveis em $90 \%$ pela polinização de vegetais nativos. No estado do Maranhão, Melipona fasciculata Smith (tiúba) já vem sendo cultivada há séculos pela população indígena, para produção de mel. Atualmente são criadas comercialmente em agrupamentos de colônias, denominados meliponários, 
que constituem a meliponicultura (Kerr, 1987; NogueiraNeto, 1997).

Melipona fasciculata Smith coleta material resinoso das plantas e traz para sua colméia, mistura com cera e barro ou terra formando o geoprópolis (Kerr, 1987), diferentemente da própolis produzida pela Apis mellifera, abelha introduzida no Brasil. Dentre os produtos apícolas a própolis vem se destacando tanto pelas suas propriedades terapêuticas como antimicrobiana, antiinflamatória, analgésica, cicatrizante, anticariogênica e anestésica, quanto pela possibilidade de aplicação na indústria alimentícia e farmacêutica (Ghisalberti, 1979; Bankova et al., 1989; Park et al., 1998a,b; Koo et al., 1999; Reis et al., 2000; Dos Santos et al., 2003; Soares et al., 2006; Longhini et al., 2007; Simões et al., 2008).

Os efeitos terapêuticos da própolis têm sido atribuídos aos diversos compostos polifenólicos que a compõem. Entre eles, os flavonóides e ácidos fenólicos podem ser considerados os principais compostos (Marcucci, 1995; Sousa et al., 2007; Lustosa et al., 2008; Nascimento et al., 2008). Silva et al. (2006) demonstraram a correlação entre os níveis de compostos fenólicos de extratos de própolis brasileiras e suas atividades antimicrobianas.

Estudos sobre composição química e ação farmacológica de geoprópolis tropical são excassos. Tomas-Barberan et al. (1993) descreveram compostos fenólicos de geoprópolis de cinco espécies de abelhas da Venezuela. Bankova et al. (1998) identificaram mais de cinqüenta compostos, principalmente fenólicos e terpênicos de geoprópolis brasileiro, produzido pelas abelhas Melipona compressipes, Melipona quadrifasciata anthidioides e Tetragona clavipes.

O estado do Maranhão apresenta um forte potencial apícola e meliponícula mas, pesquisas científicas relacionadas aos temas são incipientes, o que justifica o estudo das características físico-

químicas, químicas e farmacológicas do geoprópolis maranhense. Nogueira et al. (2004a,b) demonstraram que o geoprópolis maranhense tem cheiro, odor e sabor característico, é rico em substâncias químicas das classes dos compostos fenólicos e dos triterpenos. Gomes et al. (2003) demonstraram as atividades antiinflamatória e analgésica do extrato hidroalcoólico de geoprópolis da tiúba. Duailibe (2004) demonstrou que o uso de bochecho da solução do extrato alcoólico de geoprópolis da tiúba diminuiu em 48,5\% o número de colônias de Streptococcus mutans, na saliva dos pacientes.

O governo do estado do Maranhão inseriu nos últimos anos a apicultura e a meliponicultura dentro dos arranjos produtivos como áreas estratégicas, para desenvolver e alavancar a agricultura familiar do estado (BNB, 2001). Essas atividades são consideradas, hoje, uma das grandes opções para a região nordestina, especialmente no estado do Maranhão, por sua posição geográfica, por possuir grande número de ecossistemas, por explorar o potencial nativo da flora e por gerar trabalho e renda ao homem do campo.

A caracterização e a padronização química do geoprópolis, levando em conta as condições ambientais em que são produzidos, são fundamentais para melhorar a qualidade desses produtos e dar garantia às pessoas que os compram e os consomem.

No presente trabalho objetivou-se caracterizar do ponto de vista farmacognóstico o geoprópolis de Melipona fasciculata (tiúba), coletados em meliponários nos municípios de Arari, São Bento e São João Batista, localizados na região da Baixada maranhense, procurando estabelecer parâmetros sensoriais, perfis cromatográficos e teores de flavonóides, como base para obtenção de dados para o controle de qualidade e

Tabela 1. Características sensoriais do geoprópolis.

\begin{tabular}{rllll}
\hline Amostra & Município & Aroma & Cor & Sabor \\
\hline 1 & Arari & Inodoro & Marrom escuro & Amargo \\
2 & Arari & Inodoro & Marrom escuro & Amargo \\
3 & Arari & Inodoro & Marrom escuro & Amargo \\
4 & Arari & Inodoro & Marrom escuro & Amargo \\
5 & Arari & Inodoro & Marrom escuro & Amargo \\
6 & São João Batista & Inodoro & Marrom escuro & Amargo \\
7 & São Bento & Inodoro & Marrom escuro & Amargo \\
\hline
\end{tabular}

Tabela 2. Características sensoriais dos extratos hidroalcoólicos de geoprópolis.

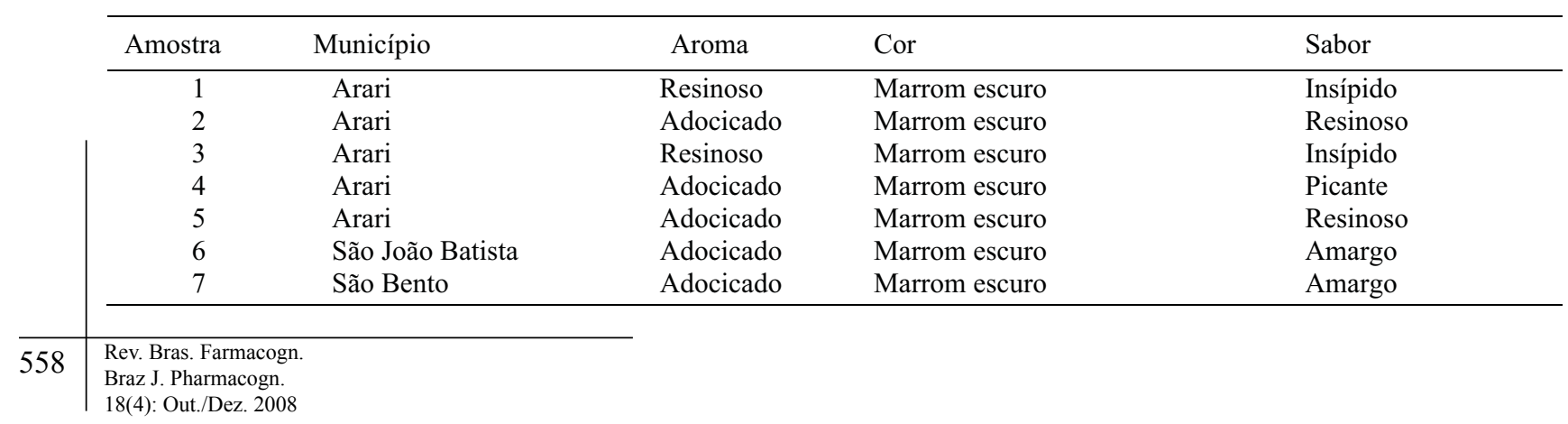


Tabela 3. Rendimento do processo extrativo dos extratos hidroalcoólicos de geoprópolis.

\begin{tabular}{ccc}
\hline Amostra & $\begin{array}{c}\text { Extrato seco de } \\
\text { geoprópolis }(\mathrm{g})\end{array}$ & $\begin{array}{c}\text { Rendimento } \\
(\%)\end{array}$ \\
\hline 1 & 1,97 & 1,36 \\
2 & 11,80 & 5,83 \\
3 & 2,30 & 1,36 \\
4 & 1,95 & 1,53 \\
5 & 12,54 & 1,10 \\
6 & 8,44 & 2,04 \\
7 & 20,10 & 2,68 \\
\hline
\end{tabular}

padronização do produto.

\section{MATERIAL E MÉTODOS}

\section{Coleta de material}

As amostras de geoprópolis de Melipona fasciculata Smith, foram coletadas com auxílio de espátulas esterilizadas, diretamente em meliponários de diferentes localidades dos municípios de Arari, São João Batista e São Bento, da Baixada maranhense, Brasil, no final da estação chuvosa e início da estação seca, durante o mês de agosto de 2003. Foram acondicionados, isoladamente em frascos de vidro âmbar esterilizados e mantidos sob refrigeração, as quais foram denominadas 1-7 respectivamente.

Os municípios da Baixada maranhense são formados por diferentes ecossistemas, como manguezais, campos inundáveis, lagoas, babaçuais e florestas (Moura, 2004).

\section{Análises sensoriais}

As amostras de geoprópolis após coleta foram submetidas a análises sensoriais de: aroma, cor e sabor (Marcucci et al., 1998; Brasil, 2001).

\section{Preparação dos extratos orgânicos de geoprópolis}

Os geoprópolis de cada localidade separadamente foram triturados em turbolizador e macerados em etanol a $70 \%$, por $24 \mathrm{~h}$, filtrados para separação da parte inorgânica (terra), obtendo-se soluções hidroalcoólicas de geoprópolis, as quais foram concentradas em evaporador rotativo e seco até peso constante. Os extratos hidroalcoólicos de geoprópolis (EHAG) foram acondicionados em frascos âmbar e mantidos sob refrigeração para as posteriores análises.

\section{Abordagem química dos EHAG}

Os EHAG foram submetidos à abordagem química para verificação de compostos das classes dos compostos fenólicos, alcalóides, terpenos e saponinas (Matos, 1997).

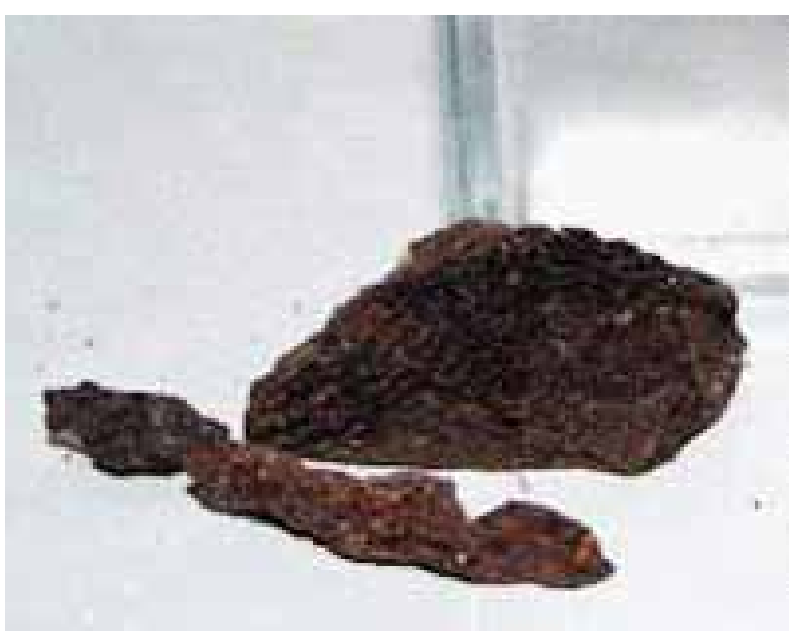

Figura 1. Geoprópolis de Melipona fasciculata Smith (tiúba).

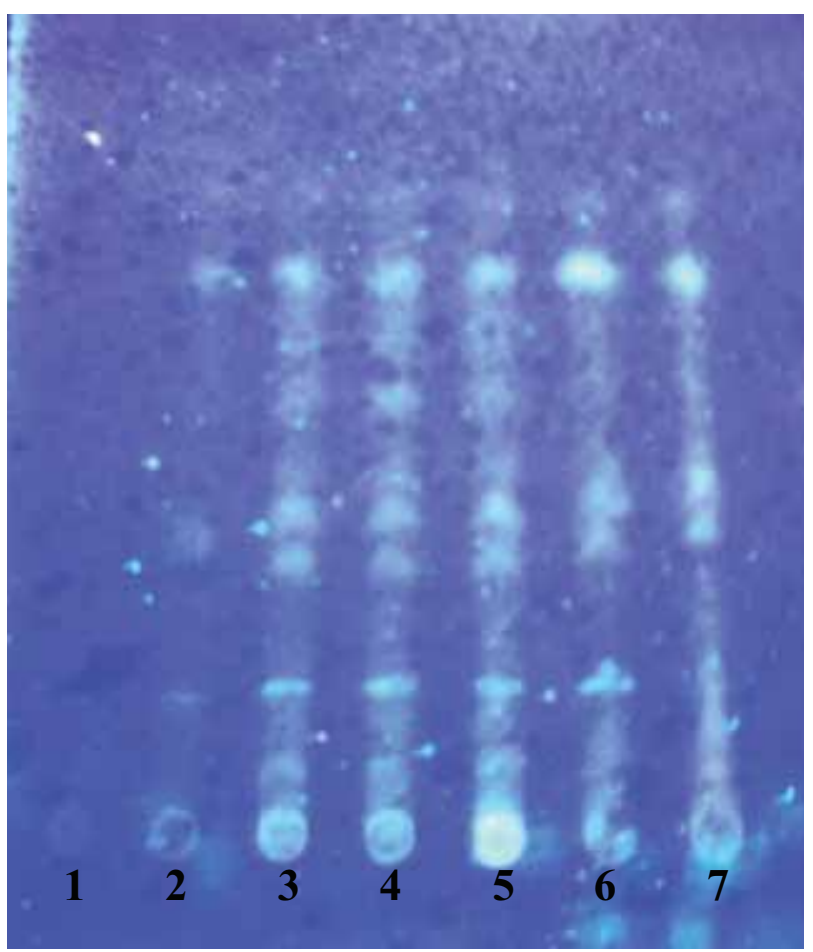

Figura 2. Cromatograma dos EHAG (1-7) de geoprópolis de Melipona fasciculata Smith (tiúba) dos municípios da Baixada Maranhense. Alíquota aplicado: $5 \mu \mathrm{L}$. Fase móvel:hexano/acetato de etila 6:4; Fase estacionária: sílicagel $60 \mathrm{~F}_{254+366}$; visualização/ revelação: luz $\mathrm{UV}_{254 \mathrm{~nm} \text { e 366nm }}$ + solução alcoólica de $\mathrm{AlCl}_{3} 1 \%$.

\section{Perfil cromatográfico dos EHAG}

Os EHAG $(5 \mu \mathrm{L})$ foram submetidos à cromatografia em camada delgada comparativa (CCDC), utilizando-se placas sílica gel $60 \mathrm{GF}_{254+366}$ (Merck), usando hexano-acetato de etila $(60: 40, \mathrm{v} / \mathrm{v})$ como fase móvel. A visualização das manchas foi efetuada por exposição da placa à luz UV $(254$ e $366 \mathrm{~nm})$. A revelação dos flavonóides, foi realizada através de borrificação com solução alcoólica de $\mathrm{AlCl}_{3} 1 \%$ (Wagner \& Bladt, 1996). As placas foram documentadas por fotografia 
Tabela 4. Teor de material inorgânico (terra) nas amostras de geoprópolis.

\begin{tabular}{clccc}
\hline Amostra & Município & Geoprópolis $(\mathrm{g})$ & $\begin{array}{c}\text { Material } \\
\text { Inorgânico(g) }\end{array}$ & $\begin{array}{c}\text { Teor da parte } \\
\text { Inorgânica(\%) }\end{array}$ \\
\hline 1 & Arari & 144,80 & 142,83 & 98,63 \\
2 & Arari & 1915,00 & 1803,20 & 94,16 \\
3 & Arari & 168,50 & 166,20 & 98,63 \\
4 & Arari & 127,30 & 125,35 & 98,46 \\
5 & Arari & 1131,90 & 1119,35 & 98,89 \\
6 & São João Batista & 413,00 & 404,56 & 97,95 \\
7 & São Bento & 747,70 & 727,60 & 97,30 \\
\hline
\end{tabular}

Tabela 5. Abordagem química dos extratos hidroalcoólicos de geoprópolis.

\begin{tabular}{ccccc}
\hline Amostra & Compostos Fenólicos & Triterpenos & Saponinas & Alcalóides \\
\hline 1 & +++ & + & + & - \\
2 & +++ & + & + & - \\
3 & +++ & - & - & - \\
4 & ++ & - & - & - \\
5 & + & + & + & - \\
6 & + & + & + & - \\
7
\end{tabular}

Nota: (+) Presença; ++ + fortemente positivo; ++ positivo; + fracamente positivo; (-) ausência.

Tabela 6. Teor de flavonóides dos extratos hidroalcoólicos de geoprópolis.

\begin{tabular}{clc}
\hline Amostra & Município & Flavonóides (\%) $^{\mathrm{a}, \mathrm{b}}$ \\
\hline 1 & Arari & $1,77 \pm 0,03$ \\
2 & Arari & $2,67 \pm 0,01$ \\
3 & Arari & $0,17 \pm 0,01$ \\
4 & Arari & $0,17 \pm 0,01$ \\
5 & Arari & $1,12 \pm 0,01$ \\
6 & São João Batista & $0,61 \pm 0,01$ \\
7 & São Bento & $0,61 \pm 0,01$ \\
\hline
\end{tabular}

${ }^{\text {a }}$ Resultados representam médias \pm desvio padrão $(n=3)$.

${ }^{\mathrm{b}}$ Expressos como equivalente de quercetina.

digital.

\section{Determinação da concentração total de flavonóides dos EHAG}

A concentração de flavonóides foi determinada utilizando $\mathrm{AlCl}_{3}$ para formar o complexo, usando espectrofotômetro Cary 50 UV-VIS a $425 \mathrm{~nm}$. Concentrações de quercetina (Merck) foram usadas como padrões e os resultados obtidos foram expressos como grama $(\mathrm{g})$ de quercetina por $10 \mathrm{~mL}$ de extrato hidroalcoólico de geoprópolis (\%) (Woisky \& Salatino, 1998; Chaillou et al., 2004; Funari \& Ferro, 2006).

\section{Análise estatística}

Todas as análises espectrofotométricas foram realizadas em triplicatas. Com esses resultados foi feita Análise de Variância (ANOVA) seguida do teste de comparações múltiplas (Newman-Keuls) utilizando o software Graph Pad Prism, versão 4.03, tomando-se o valor de $p \leq 0,05$ como nível máximo de significância estatística (Sokal \& Rohlf, 1996).

\section{RESULTADOS E DISCUSSÃO}

Os geoprópolis apresentaram-se como fragmentos rígidos de diferentes tamanhos, com granulometria heterogênea (Figura 1), inodoros, coloração marrom escuro e sabor amargo, conforme dados da Tabela 1. Constatou-se que a predominância de terra nas amostras não permite uma diferenciação sensorial.

As análises sensoriais dos EHAG estão descritos na Tabela 2. As amostras 1-5 possuem cor marrom escuro, odor resinoso a adocicado e sabor insípido, enquanto as amostras 6-7 apresentam odor adocicado, sabor amargo e cor marrom escuro. Esses caracteres são similares aos encontrados para a própolis de Apis mellifera, coletadas em diferentes regiões brasileiras (Marcucci, 1995; Park et al., 2002; Franco et al., 2000; Funari \& Ferro, 2006).

Os rendimentos dos processos extrativos das amostras de geoprópolis obtidos por maceração em etanol a $70 \%(\mathrm{v} / \mathrm{v})$ variaram entre $1,10-5,83 \%$ (Tabela 3). Bankova et al. (1998) submeteram amostras de geoprópolis de Melipona compressipes 
coletadas no município de Picos-PI a maceração em etanol a $70 \%(\mathrm{v} / \mathrm{v})$, obtendo rendimento de $5 \%$ de extrato seco, enquanto que amostras de geoprópolis de Melipona quadrifasciata anthidioides do município de Prudentópolis-PR, tiveram rendimento de 17\%. Estudos realizados por Cunha et al. (2004) indicam que o maior rendimento nos processos de extração de própolis por maceração ocorre nos sistemas onde a proporção de etanol é igual ou superior a $70 \%(\mathrm{v} / \mathrm{v})$.

A tiúba utiliza além do material retirado das plantas, terra para formar o geoprópolis, cujo teor variou aproximadamente entre 94-98\% (Tabela 4), diferentemente de outras espécies de Apidae, como Apis mellifera, que utilizam material das plantas e adicionam cera e secreções salivares para formar a própolis.

A abordagem química dos extratos hidroalcoólicos de geoprópolis indicou a presença de substâncias das classes dos compostos fenólicos, dos triterpenos e das saponinas (Tabela 3) predominando maior concentração de compostos fenólicos nas amostras 1-3, diminuindo a concentração dos mesmos nas amostras 5-7. Substâncias fenólicas estão presentes em própolis de Apis mellifera européias e sul-americanas (Greenaway et al., 1990; Marcucci et al., 1998; Woisky \& Salatino, 1998; Bankova et al., 2000) e em geoprópolis brasileiras das abelhas Melipona compressipes (PicosPI), Tetragona clavipes e Melipona quadrifasciata anthidioides (Prudentópolis-PR) (Tomas-Barberan et al., 1993; Bankova et al., 1998). Somente as amostras 1, 2,5 e 6 apresentaram reação fracamente positiva para triterpenos e saponinas. Substâncias triterpênicas têm sido detectadas em própolis tropicais de Apis mellifera e geoprópolis (Bankova et al., 2000; Silva et al., 2005). Não foram detectados alcalóides nas amostras analisadas.

$\mathrm{Na}$ Figura 2 estão apresentados os perfis cromatográficos dos EHAG. As amostras 1-7 analisadas por CCDC e reveladas com lâmpadas UV indicaram presença de manchas fluorescentes em cada amostra. A intensificação das fluorescências nas manchas, após borrificação com $\mathrm{AlCl}_{3} 1 \%$, comprova a presença de substâncias flavonoídicas. A presença dos flavonóides foi confirmada pelo doseamento dos mesmos utilizando ensaios espectrofotométricos.

Os teores de flavonóides encontrados nas amostras variaram entre 0,17 - 2,67\% (Tabela 6). Constatou-se que a amostra 2 apresenta o maior teor de flavonóides (2,67\%), que de acordo com a legislação brasileira para classificação de própolis de Apis mellifera, pode ser considerada como de alto teor de flavonóides. As amostras 1 (1,77\%), 5 (1,12\%), 6 e $7(0,61 \%)$, apresentaram valores acima do teor médio exigido pela legislação. Enquanto as amostras 3 e 4 apresentaram $0,17 \%$ de flavonóides, podendo ser classificadas de própolis de baixo teor, conforme exigido pela referida legislação (Brasil, 2001; Funari \& Ferro, 2006). Ressalta-se a falta de legislação para produtos de outras espécies de abelhas.

Os resultados da análise estatística, evidenciaram diferenças significativas entre os EHAG $1,2,5,6$ e 7 para o teor de flavonóides $(p<0,001)$, entretanto entre os EHAG 3 e 4 a diferença não foi significativa $(p \geq 0,05)$.

A variação qualitativa e quantitativa de flavonóides e outros constituintes químicos em própolis e geoprópolis é fortemente afetadas pela flora visitada pelas abelhas, região geográfica e fatores climáticos (Teixeira et al., 2003; Bankova, 2005; Gomes-Caravaca et al., 2006). Considerando que a região da Baixada maranhense é formada por ecossistemas heterogêneos, constituindo-se de litoral atlântico, reentrâncias e manguezais, lagoas e terras alagadas, terrenos altos com capoeiras, babaçuais e mata (Moura, 2004), é justificada a variação destes constituintes químicos.

Os resultados obtidos permitem obter parâmetros de controle qualidade para o geoprópolis e seus derivados da Baixada maranhense. Considerando que a região é uma grande produtora de mel, produto base do negócio apícola e meliponícula, o conhecimento do perfil de qualidade agrega valores a mais um produto deste setor, contribuindo para o desenvolvimento do agronegócio no estado do Maranhão.

\section{AGRADECIMENTOS}

Ao $\mathrm{CNPq}$ pelo auxílio financeiro (Proc $\mathrm{n}^{\circ}$ CNPq/UEMA 5507387/01-0) e bolsa de Iniciação Cientifica para Alexandre M. C. Nogueira. À FAPEMA pela bolsa de mestrado de Richard Pereira Dutra. A Central Analítica do Departamento de Química - UFMA pelo uso do espectrofotômetro UV-VIS.

\section{REFERÊNCIAS}

Bankova V, Popov S, Marekov NL 1989. Isopentenyl cinnamates from poplar buds and propolis. Phytochemistry 28: 871-873.

Bankova V, Christov R, Marcucci MC, Popov S 1998. Constituents of brazilian geopropolis. $Z$ Naturforschung 53c: 402-406.

Bankova V, Castro SL, Marcucci MC 2000. Propolis: recent advances in chemistry and plant origin. Apidologie 31: 3-15.

Bankova V 2005. Chemical diversity of propolis and the problem of standardization. J Ethnopharmacol 100: 114-117.

BNB 2001. Diversificação da produção Apícola. Palestra proferida no lançamento do programa de desenvolvimento da apicultura do Estado do Maranhão. Banco do Nordeste, São Luís, Brasil.

Brasil 2001. Ministério de Agricultura e do Abastecimento. Instrução normativa $\mathrm{n}^{\circ} 3$ - Anexo VI - Regulamento técnico para fixação de identidade e qualidade de própolis. Diário Oficial da Republica Federativa do Brasil. Brasília, Brasil.

Chaillou LL, Herrera HA, Maidana JF 2004. Estudo de própolis de Santiago Del Estero, Argentina. Cien Tecnol Aliment 24: 11-15. 
Cunha IBS, Sawaya ACHF, Caetano FM, Shimizu MT, Marcucci MC, Drezza FT, Povia GS, Carvalho PO 2004. Factors that influence the yield and composition of Brazilian propolis extracts. J Braz Chem Soc 15: 964-970.

Dos Santos CR, Arcenio F, Carvalho ES, Lúcio EMRA, Araújo GL, Teixeira LA, Sharapin N, Rocha L 2003. Otimização do processo de extração de própolis através da verificação da atividade antimicrobiana. Rev Bras Farmacogn 13: 71-74.

Duailibe SAC 2004. Efeito do extrato de própolis na contagem de Streptococcus mutans da cavidade oral. São Luís, 73 p. Dissertação de Mestrado - Programa de PósGraduação em Ciências da Saúde, Universidade Federal do Maranhão.

Franco SL, Bruschi ML, Moura LPP, Bueno JHF 2000. Avaliação farmacognóstica da própolis da região de Maringá. Rev Bras Farmacogn 9/10: 1-10.

Funari CS, FerroVO 2006. Análise de própolis. Cien Tecnol Aliment 26: 171-178.

Ghisalberti EL 1979. Propolis: A review. Bee World 60: 5984.

Gomes VA, Nogueira AMC, Marques EC, Santana ER, Barros FER, Borges MOR, Borges ACR, Ribeiro MNS 2003. Estudos das atividades antiinflamatórias e antinociceptiva da própolis de Melipona compressipes fasciculata Smith (tiúba). XV Seminário de Iniciação Cientifica. São Luís, Brasil.

Gómez-Caravaca AM, Gómez-Romero M, Arráez-Román D, Segura-Carretero A, Fernández-Gutiérrez A 2006. Advances in the analysis of phenolic compounds in products derived from bees. J Pharmacol Biom Anal 41: $1220-1234$

Greenaway W, Scaysbrook T, Whatley FR 1990. The composition and plant origins of propolis. Bee World 71: 107-118.

Kerr W 1987. Abelhas indígenas brasileiras (meliponíneos) na polinização e na produção de mel, pólen, geoprópolis e cera. Inf Agropec 13: 15-27.

Koo H, Park YK, Ikegaki M, Cury JA, Rosalen PL 1999. Effect of Apis mellifera propolis from two Brazilian regions on caries development in desalivated rats. Caries Res 33: 393-400.

Longhini R, Raksa SM, Oliveira ACP, Svidzinski TIE, Franco SL 2007. Obtenção de extratos de própolis sob diferentes condições e avaliação de sua atividade antifúngica. Rev Bras Farmacogn 17: 388-395.

Lustosa SR, Galindo AB, Nunes LCC, Randau KP, Rolim Neto PJ 2008. Própolis: atualizações sobre a química e a farmacologia. Rev Bras Farmacogn 18: 447-454.

Marcucci MC 1995. Própolis: a chemical composition, biological properties and therapeutic activity. Apidologie 26: 83-99.

Marcucci MC, Rodriguez J, Ferreres F, Bankova V, Groto R, Popov S 1998. Chemical composition of Brazilian propolis from São Paulo State. Z Naturforsch $53 c$ : 117-119.

Matos FJA 1997. Introdução à Fitoquímica Experimental. Fortaleza: Edições UFC.

Moura EG 2004. Agroambientes de Transição: entre o trópico úmido e o semi-árido do Brasil. São Luís: UEMA.

Nascimento EA, Chang R, Morais SAL, Piló-Veloso D, Reis DC 2008. Um marcador químico de fácil detecção para a própolis de Alecrim-do-Campo (Baccharis dracunculifolia). Rev Bras Farmacogn 18: 379-386.

Nogueira AMC, Matos RRO, Costa MCP, Ribeiro MNS 2004a. Própolis da tiúba (Melipona compressipes fasciculata Smith): aspectos químicos e físico-químicos. $L V I$
Reunião Anual da Sociedade Brasileira Progresso da Ciência. Brasília, Brasil.

Nogueira, AMC, Matos RRO, Gomes VA, Ribeiro MNS, Borges MO R, Borges ACR, Costa MCP 2004b. Química e farmacologia de geoprópolis da tiúba. $I V$ Reunião Regional da Sociedade Brasileira para o Progresso da Ciência. São Luís, Brasil.

Nogueira-Neto P 1997. A vida e criação de abelhas indigenas sem ferrão (Meliponinae). São Paulo: Nogueirapes.

Park YK, Koo H, Ikegaki M, Cury JA, Rosalen PL, Abreu JAS 1998a. Antimicrobial activity of propolis on oral microorganisms. Cur Microbiol 34: 24-28.

Park YK, Koo H, Ikegaki M, Cury JA, Rosalen PL, Abreu JAS 1998b. Effect of propolis on Streptococcus mutans, Actinomyces naeslundii and Staphylococcus aureus. Rev Microbiol 29: 143-148.

Park YK, Alencar SM, Aguiar CL 2002. Botanical origin and chemical composition of Brasilian propolis. J Agric Food Chem 50: 2502-2506.

Reis CMF, Carvalho JCT, Caputo LRG, Patrício KCM, Barbosa MVJ, Chieff AL, Bastos JV 2000. Atividade antiinflamatória, antiúlcera gástrica e toxicidade subcrônica do extrato etanólico de própolis. Rev Bras Farmacogn 9/10: 43-52.

Silva MSS, Cito AMGL, Chaves MH, Lopes JAD 2005. Triterpenóides tipo cicloartano de própolis de Teresina - PI. Quim Nova 28: 801-804.

Silva JFM, Souza MC, Matta SR, Andrade MR, Vidal FVN 2006. Correlation analysis between phenolic levels of Brazilian propolis extracts and their antimicrobial and antioxidant activities. Food Chem 99: 431-435.

Simões CC, Araújo DB, Araújo RPC 2008. Estudo in vitro e ex vivo da ação de diferentes concentrações de extratos de própolis frente aos microrganismos presentes na saliva de humanos. Rev Bras Farmacogn 18: 84-89.

Soares AKA, Carmo GC, Quental DP, Nascimento DF, Bezerra FAF, Moraes MO, Moraes MEA 2006. Avaliação da segurança clínica de um fitoterápico contendo Mikania glomerata, Grindelia robusta, Copaifera officinalis, Myroxylon toluifera, Nasturtium officinale, própolis e mel em voluntários saudáveis. Rev Bras Farmacogn 16: 447-454.

Sokal RR, Rohlf FJ 1996. Biometry: The principle and practice of statistics. Biological Research. 2 ed. New York: W.H. Freeman and company.

Sousa JPB, Furtado NAJC, Jorge R, Soares AEE, Bastos JK 2007. Perfis físico-químico e cromatográfico de amostras de própolis produzidas nas microrregiões de Franca (SP) e Passos (MG), Brasil. Rev Bras Farmacogn 17: 85-93

Teixeira EW, Message D, Meira RMSA, Salatino A 2003. Indicadores da origem botânica da própolis: Importância e perspectivas. Boletim da Industria Animal, Nova Odessa 60: 83-106.

Tomas-Barberan FA, Garcia-Viguera C, Vit-Olivier P, Ferreres F, Tomas-Lorente F 1993. Phytochemical evidence for the botanical origin of tropical from Venezuela. Phytochemistry 34: 191-196.

Wagner H, Bladt S 1996. Plant drug analysis: a thin layer chromatography atlas. Berlin: Springer.

Woisky RGR, Salatino A 1998. Analysis of propolis: some parameters and procedures for chemical quality control. J Apic Res 37: 99-105. 